\title{
Weight Loss and Impact on Quality of Life in Parkinson's Disease
}

\author{
Umer Akbar ${ }^{1}$, Ying $\mathrm{He}^{2}$, Yunfeng $\mathrm{Dai}^{3}$, Nawaz $\mathrm{Hack}^{4}$, Irene Malaty ${ }^{4}$, Nikolaus \\ R. McFarland ${ }^{4}$, Christopher Hess ${ }^{4}$, Peter Schmidt ${ }^{5}$, Samuel $\mathrm{Wu}^{3}$, Michael S. Okun ${ }^{4}$ \\ 1 Department of Neurology, Brown University, Providence, Rhode Island, United States of America, \\ 2 Department of Mathematics, Clarkson University, Potsdam, New York, United States of America, \\ 3 Department of Biostatistics, University of Florida, Gainesville, Florida, United States of America, \\ 4 Department of Neurology, University of Florida, Gainesville, Florida, United States of America, 5 National \\ Parkinson Foundation, Miami, Florida, United States of America \\ *umerakbar@yahoo.com
}

\section{G openaccess}

Citation: Akbar U, He Y, Dai Y, Hack N, Malaty I, McFarland NR, et al. (2015) Weight Loss and Impact on Quality of Life in Parkinson's Disease. PLoS ONE 10(5): e0124541. doi:10.1371/journal.pone.0124541

Academic Editor: Alfonso Fasano, University of Toronto, CANADA

Received: July 11, 2014

Accepted: March 15, 2015

Published: May 4, 2015

Copyright: $\odot 2015$ Akbar et al. This is an open access article distributed under the terms of the Creative Commons Attribution License, which permits unrestricted use, distribution, and reproduction in any medium, provided the original author and source are credited.

Data Availability Statement: Data are from the National Parkinson Foundation quality improvement initiative. Data can be made available by contacting Peter Schmidt (director of NPF) at

pschmidt@parkinson.org.

Funding: The authors received no specific funding for this work.

Competing Interests: Dr. Okun serves as a consultant for the National Parkinson Foundation, and has received research grants from NIH, NPF, the Michael J. Fox Foundation, the Parkinson Alliance, Smallwood Foundation, the Bachmann-Strauss Foundation, the Tourette Syndrome Association, and

\section{Abstract}

\section{Introduction}

Weight loss is common in Parkinson's Disease (PD) and sometimes may precede the diagnosis. Weight loss is associated with multiple factors but its impact on health-related quality of life (HRQL) in PD remains unknown. We sought to investigate the factors associated with weight change and to quantify its effect on HRQL.

\section{Methods}

The National Parkinson Foundation Quality Improvement Initiative (NPF-QII) data was used to analyze PD patients longitudinally between two visits, separated by $12 \pm 6$ months. Multiple linear regression analyses were used to assess the associations between baseline covariates and body weight change per month, and to evaluate whether, and to what degree, Parkinson's Disease Questionnaire (PDQ-39) scores were affected.

\section{Results}

A higher Hoehn \& Yahr stage, higher number of comorbidities, older age, lower MOCA estimate, and higher rate of levodopa usage were observed in patients who lost weight. Multivariate regression analysis indicated that age and levodopa usage were significantly associated with weight loss. Furthermore, monthly body weight loss was significantly associated with $\mathrm{HRQL}$ decline in PD patients. Loss of $1 \mathrm{lb}(0.45 \mathrm{~kg})$ per month was associated with a decline in QOL: an increase of $0.5 \%$ in PDQ-39 Summary Index score $(p=0.004)$, and $1.1 \%$ and $1.5 \%$ increases in the mobility and ADL dimensions, respectively.

\section{Conclusion}

Weight loss in PD is common and seems to correlate with worsened HRQL. Awareness of factors associated with weight loss and its relation to HRQL may help practitioners improve patient management and expectations. 
the UF Foundation. Dr. Okun has received royalties for publications with Demos, Manson, Amazon, Smashwords, and Cambridge (movement disorders books). This does not alter the authors' adherence to PLOS policy on sharing data and materials.

\section{Introduction}

Parkinson's disease (PD) is a chronic progressive neurodegenerative syndrome that impacts both motor and non-motor domains.[1] Treatment of PD is largely symptomatic and much of the focus has been on improvement of function and health-related quality of life (HRQL).[1] PD treatment frequently targets the motor symptoms including tremor, rigidity, slowness, and gait impairment. However, there are many non-motor features that frequently emerge, including impaired sensory perception (anosmia, dysgeusia, tingling and pain), mood changes (depression, anxiety), cognitive decline (executive dysfunction, slowed thinking), dysautonomia (constipation, orthostatic hypotension, erectile dysfunction), and apathy (with or without depression).[2] These symptoms may individually, or in combination, lead to a decreased food intake or, alternatively, to an increased energy expenditure with the possibility of consequent weight loss.[3-6] In addition, the neurodegenerative process itself may affect regions of the brain important to the maintenance of weight (e.g. hypothalamus).[7]

Weight loss is a common feature of $\mathrm{PD}$, and may predate expert diagnosis. Chen et al.[3] observed that in the 10 years preceding a PD diagnosis, the mean body weight decreased by 5.2 pounds (lbs) Although body weight was unchanged 2 to 4 years prior to PD diagnosis, weight decreased by 7.7 lbs over the next 8 years. Additionally, PD stage has also been correlated to weight loss. [8] The current study asks an important question about weight loss in PD, and whether it correlates with HRQL.

We sought to examine PD weight loss and its association with HRQL. Using a prospectively compiled longitudinal PD outcomes database, we quantified the effect of the mean monthly PD weight loss and analyzed the consequent impact on HRQL. Our hypothesis was that patients with more advanced disease would have lower body weight and worse HRQL. We further hypothesized that longitudinal weight loss would adversely affect HRQL.

\section{Methods}

\section{Data source}

The National Parkinson Foundation (NPF) prospectively compiles and maintains long-term clinical outcomes of people with PD drawn from fifteen Centers of Excellence within and outside of the United States as part of the NPF Quality Improvement Initiative clinical study (NPF-QII). Patients who consent to enrollment are annually examined in-person. The outcomes project collects demographic information, disease onset, duration, stage and symptomseverity, living conditions, comorbid conditions, medications, other treatments/referrals, and clinical condition, as well as outcomes of several validated measures including the timed upand-go (TUG) test, several cognition measures, the Parkinson's Disease Questionnaire [9] (PDQ-39), and multi-dimensional caregiver strain. Since the NPF-QII data is de-identified, a full review by the institutional review board was not required.

\section{Subject selection \& definitions}

The design was a retrospective cohort study using the data prospectively collected from the NPF-QII longitudinal dataset. All PD patients with two subsequent visits separated by 12 months ( \pm 6 months) were included. The variables analyzed included age, gender, age at onset, disease duration, living situation, presence of a regular care partner, Hoehn \& Yahr (H \& Y) stage, presence of rest tremor, motor fluctuations, TUG test, immediate and delayed word recall, verbal fluency, MOCA estimate, number of comorbidities, usage of levodopa, antidepressants, antipsychotics, speech therapy, exercise, social worker utilization, and mental health 
referral. HRQL was measured by a validated scale, the PDQ-39. The PDQ-39 Summary Index score (PDQ-SI), as well as its ADL and mobility dimensions, were analyzed.

As part of the data collection process, the NPF-QII instructs examining clinicians to estimate their diagnostic certainty of PD as being more than $90 \%, 50-90 \%$, or less than $50 \%$. Only subjects who were classified as having PD with greater than $90 \%$ certainty were included for analysis.

The $25^{\text {th }}$ percentile of absolute weight change was less than $2 \mathrm{lbs}(0.9 \mathrm{~kg})$, which was used as cutoff of "no change". Subjects who lost $\geq 2 \mathrm{lbs}(0.9 \mathrm{~kg})$ were compared to subjects who lost $<2$ lbs or had no change. In addition, we treated patients whose weight change was more than 3 standard deviations away from the sample mean as outliers. Their body weight may have been erroneously entered, or caused by a medical comorbidity (e.g., malignancy).

Collection of data was conducted under the approval of the University of Florida IRB-01 (approval \#308-2009). Patient records in the NPF-QII data were de-identified prior to analysis.

\section{Statistical analysis}

A cross-sectional analysis was conducted for PD patients at the first visit. Baseline demographic and clinical characteristics were compared between patients who lost weight versus those who gained weight or had no change. The analysis of variance for continuous variables (ANOVA) and a chi-square test or a fisher-exact test for categorical variables were utilized. The effect of weight change on HRQL was analyzed longitudinally for patients with available data at visits 1 and 2. "Monthly weight change" was calculated by dividing the change in body weight (in pounds) by the follow-up interval (in months). For patients with available data at the follow-up visit (12 \pm 6 months), multiple linear regression analyses were used to assess the associations between baseline covariates and body weight change per month. Further, the impact of monthly weight change on HRQL measures (PDQ-SI score and ADLs and mobility dimensions) was analyzed while adjusting for covariates selected from a list of 18 pre-specified factors (listed above). Commercially available SAS software (version 9.2) was used to perform the statistical analysis. All tests were two-sided and p-values less than 0.05 were deemed statistically significant.

\section{Results}

A total of 5443 PD patients had first visit data available at the time of the data request and 4633 patients were diagnosed with idiopathic PD with reported greater than $90 \%$ certainty by a movement disorder specialist at one of the NPF Centers of Excellence. The analyses included 1718 patients, after exclusion of those patients with no data available at a second visit $(\mathrm{n}=2476)$, those whose follow-up visit date was not between 6 and 18 months from the first visit $(\mathrm{n}=355)$, and 84 patients with no weight data or with a weight change greater than three standard deviations (mean $28 \mathrm{lb}$ or $12.7 \mathrm{~kg}$ ).

Baseline clinical characteristics, demographic data, and social variables are presented in Table 1. A higher Hoehn \& Yahn stage, higher number of comorbidities, older age, lower MOCA estimate, and higher rate of levodopa usage were observed in patients who lost weight more than $2 \mathrm{lbs}(0.9 \mathrm{~kg})$ as compared to patients who gained weight or had no change. Furthermore, based on multivariate regression analysis, we found that older age and levodopa usage were associated with larger weight loss per month. Specifically, when age was controlled, patients on levodopa lost approximately $0.18 \mathrm{lb}(0.08 \mathrm{~kg})$ per month more than patients not on levodopa ( $\mathrm{p}=0.007)$; and controlling for levodopa use, a ten year increase in age was associated with a mean loss of $0.08 \mathrm{lb}(0.04 \mathrm{~kg})$ per month $(\mathrm{p}=0.001)$. 
Table 1. Demographic, clinical and social variables.

\begin{tabular}{|c|c|c|c|c|c|}
\hline & & All subjects & Subjects with weight loss* & Subjects with weight gain or no change & P-value \\
\hline & & $(n=1718)$ & $(n=757)$ & $(n=961)$ & \\
\hline \multirow[t]{4}{*}{ Hoehn \& Yahr stage } & $1-1.5$ & 189 & $75(10.3 \%)$ & $114(12.3 \%)$ & 0.013 \\
\hline & $2-2.5$ & 935 & $402(55.1 \%)$ & $533(57.5 \%)$ & \\
\hline & $3-3.4$ & 446 & $222(30.5 \%)$ & $224(24.2 \%)$ & \\
\hline & 4 or over 4 & 86 & $30(4.1 \%)$ & $56(6.0 \%)$ & \\
\hline \multicolumn{2}{|c|}{ Standardized TUG** } & $-0.18 \pm 0.95$ & $-0.19 \pm 0.95$ & $-0.18 \pm 0.95$ & 0.776 \\
\hline \multicolumn{2}{|c|}{ Antidepressant medications used } & 501 & $226(30.1 \%)$ & $275(28.7 \%)$ & 0.553 \\
\hline \multicolumn{2}{|c|}{ Number of comorbidities } & $1.7 \pm 1.3$ & $1.8 \pm 1.3$ & $1.6 \pm 1.3$ & 0.002 \\
\hline \multicolumn{2}{|c|}{ Age } & $66.3 \pm 9.6$ & $67.5 \pm 9.4$ & $65.3 \pm 9.7$ & $<.0001$ \\
\hline \multicolumn{2}{|c|}{ Disease duration } & $9.2 \pm 6.0$ & $9.3 \pm 5.9$ & $9.1 \pm 6.1$ & 0.364 \\
\hline \multicolumn{2}{|c|}{ Moca estimate } & $24.4 \pm 3.3$ & $24.1 \pm 3.3$ & $24.7 \pm 3.3$ & 0.002 \\
\hline \multicolumn{2}{|c|}{ Social worker/counseling } & 200 & $93(12.3 \%)$ & $107(11.1 \%)$ & 0.465 \\
\hline \multicolumn{2}{|c|}{ Presence of Motor fluctuations } & 805 & $369(49.1 \%)$ & $436(45.6 \%)$ & 0.142 \\
\hline \multicolumn{2}{|c|}{ Antipsychotic medications } & 74 & $37(4.9 \%)$ & $37(3.9 \%)$ & 0.285 \\
\hline \multirow[t]{5}{*}{ Regular care partner } & Spouse/partner & 224 & $107(14.2 \%)$ & $117(12.2 \%)$ & 0.742 \\
\hline & Other relative & 1392 & $603(79.9 \%)$ & $789(82.2 \%)$ & \\
\hline & Paid caregiver & 71 & $31(4.1 \%)$ & $40(4.2 \%)$ & \\
\hline & Other & 22 & $11(1.5 \%)$ & $11(1.1 \%)$ & \\
\hline & No & 6 & $3(0.4 \%)$ & $3(0.3 \%)$ & \\
\hline \multicolumn{2}{|c|}{ Mental health treatment or referral } & 162 & $65(8.6 \%)$ & $97(10.1 \%)$ & 0.289 \\
\hline \multicolumn{2}{|c|}{ Speech therapy } & 195 & $92(12.2 \%)$ & $103(10.7 \%)$ & 0.360 \\
\hline \multicolumn{6}{|c|}{ Gender } \\
\hline \multicolumn{2}{|c|}{ Male } & 1089 & $470(62.1 \%)$ & $619(64.4 \%)$ & 0.321 \\
\hline \multicolumn{2}{|c|}{ Female } & 629 & 287 (37.9\%) & $342(35.6 \%)$ & \\
\hline \multicolumn{2}{|c|}{ Levodopa usage } & 1450 & $675(89.2 \%)$ & $775(80.7 \%)$ & $<0.0001$ \\
\hline \multicolumn{2}{|c|}{ Presence of rest tremor } & 1240 & $565(75.2 \%)$ & $675(71.1 \%)$ & 0.054 \\
\hline \multicolumn{2}{|c|}{ PDQ-Mobility } & $11.3 \pm 10.4$ & $11.7 \pm 10.2$ & $11.0 \pm 10.5$ & 0.181 \\
\hline \multicolumn{2}{|c|}{ PDQ-ADL } & $6.9 \pm 5.5$ & $6.9 \pm 5.4$ & $6.9 \pm 5.6$ & 0.963 \\
\hline \multicolumn{2}{|c|}{ MCSI total } & $16.7 \pm 15.2$ & $17.3 \pm 15.5$ & $16.2 \pm 15.0$ & 0.206 \\
\hline \multicolumn{2}{|c|}{ Dopamine agonist } & 715 & 298 (39.4\%) & $417(43.5 \%)$ & 0.086 \\
\hline
\end{tabular}

* $25 \%$ of patients had less than $2 \mathrm{lb}$ absolute weight change; patients with $2 \mathrm{lb}$ or more decrease were designated as losing weight.

** Smaller standardized TUG means using less help or taking less time to stand up and go.

doi:10.1371/journal.pone.0124541.t001 
Table 2. Regression analysis of change in PDQ-39 total score.

\begin{tabular}{|c|c|c|c|c|c|c|}
\hline Variable & \multicolumn{3}{|c|}{ Full Model } & \multicolumn{3}{|c|}{ Final Model } \\
\hline Name & Estimate & Standard Error & P-Value & Estimate & Standard Error & P-Value \\
\hline Intercept & 4.950 & 6.026 & 0.412 & -1.013 & 1.089 & 0.353 \\
\hline Weight change per month & -0.769 & 0.364 & 0.035 & -0.729 & 0.311 & 0.019 \\
\hline \multicolumn{7}{|c|}{ Hoehn \& Yahr stage (reference level: 4-5) } \\
\hline 1 & -0.702 & 1.671 & 0.675 & & & \\
\hline 2 & -0.742 & 1.446 & 0.608 & & & \\
\hline 3 & -0.135 & 1.410 & 0.924 & & & \\
\hline Standardized TUG & -0.355 & 0.346 & 0.305 & & & \\
\hline Antidepressant use & -0.331 & 0.614 & 0.590 & & & \\
\hline Number of comorbidities & -0.109 & 0.216 & 0.614 & & & \\
\hline Age & 0.011 & 0.033 & 0.735 & & & \\
\hline Disease duration & 0.010 & 0.051 & 0.837 & & & \\
\hline MOCA estimate & -0.083 & 0.089 & 0.352 & & & \\
\hline $\begin{array}{l}\text { Lack of social worker/ } \\
\text { counseling }\end{array}$ & 1.357 & 0.844 & 0.108 & & & \\
\hline Presence of motor fluctuations & 0.842 & 0.588 & 0.152 & & & \\
\hline Antipsychotic medications & 1.888 & 1.351 & 0.163 & & & \\
\hline \multicolumn{7}{|c|}{ Regular care partner (Reference level: No) } \\
\hline Spouse/Partner & -3.786 & 4.596 & 0.410 & & & \\
\hline Other Relative & -3.632 & 4.547 & 0.425 & & & \\
\hline Paid Caregiver & -1.291 & 4.726 & 0.785 & & & \\
\hline Other & -4.418 & 5.208 & 0.396 & & & \\
\hline $\begin{array}{c}\text { Mental health treatment or } \\
\text { referral }\end{array}$ & -2.078 & 0.958 & 0.030 & & & \\
\hline Speech therapy & 0.950 & 0.871 & 0.275 & & & \\
\hline Male gender & -0.815 & 0.568 & 0.151 & & & \\
\hline Levodopa usage & -0.100 & 0.835 & 0.905 & & & \\
\hline Presence of rest tremor & -1.491 & 0.613 & 0.015 & -1.336 & 0.581 & 0.022 \\
\hline Dopamine agonist & -0.852 & 0.555 & 0.125 & & & \\
\hline
\end{tabular}

doi:10.1371/journal.pone.0124541.t002

\section{Discussion}

This study aimed to determine whether weight changes in PD patients correlate with clinical characteristics, social factors, and HRQL outcomes. The results reveal that interval weight loss is associated with a higher $\mathrm{H} \& \mathrm{Y}$ stage, higher number of comorbidities, older age, a lower MOCA estimate, and a higher rate of levodopa usage. We demonstrate significant correlations between interval weight loss and decline in HRQL (PDQ-SI, and mobility and ADLs dimensions).

Increasing age is one of several factors previously shown to be strongly associated with weight loss in PD.[5] Older age, worse MOCA scores and higher rates of levodopa usage are all suggestive of disease progression. In addition to the general frailty which accompanies aging, PD patients with later-onset disease progress more rapidly, and are at particular risk for becoming unable to care for themselves.[10] Furthermore, younger PD patients requiring medication are usually initiated on dopamine agonists which can precipitate compulsive eating and weight gain.[11] Our results demonstrate a trend, without statistical significance, for fewer weight-losing subjects to be on a dopamine agonist. 
In addition to monthly weight loss, two other covariates negatively affected change in HRQL: lack of referral to a mental health expert, and absence of rest tremor. In our cohort, we did not observe significant correlations between other factors previously reported, such as H\&Y and MOCA, which may relate to the potential bias of patients drawn from the expert clinicians. Controlling for the two covariates found (mental health referral and absent rest tremor), each $1 \mathrm{lb}(0.45 \mathrm{~kg})$ weight loss led to a statistically significant worsening of the PDQ-SI by $0.5 \%$, mobility dimension by $1.1 \%$, and ADL dimension by $1.5 \%$. The practical effect of these small changes in HRQL on a monthly basis may not appear meaningful, but when taken in aggregate over a longer follow-up interval, they become significant and suggest that weight loss should be carefully monitored and addressed in PD patients. For example, the loss of $5 \mathrm{lbs}$ $(2.25 \mathrm{~kg})$ noted during a patient's routine follow-up visit would be associated with an estimated decline in HRQL by $2.5 \%, 5.5 \%$ and $7.5 \%$ in PDQ-SI, mobility and ADL scores. Using this method as a tool may possibly prompt an investigation to elucidate the cause of weight loss and discuss potential management options to improve HRQL.

PD weight loss cannot be attributed entirely to any one factor.[12, 13, 3, 5] Two review articles have been published with more thorough discussions about nutritional status and weight change in PD. [12, 13] Many elements impact food intake and energy expenditure in PD, however, these factors do not completely account for weight loss. These factors may include higher $\mathrm{H} \& \mathrm{Y}$ stage, increasing age, use of levodopa, worsening cognition, development of motor fluctuations, absence of tremor, and low overall physical activity.[12, 13, 3, 5, 6] Though mainly a hypokinetic movement disorder, several features of $\mathrm{PD}$ can potentially contribute to an increased energy expenditure, including tremor, rigidity and dyskinesia. Studies of resting energy expenditure in PD have demonstrated increased calorie consumption. $[4,14,15]$ The notion that this increase in energy expenditure is the major cause of weight loss in PD is supported by the observation that weight loss precedes the diagnosis even when there is an increase in energy intake. $[3,4,14]$ This view is opposed by a study demonstrating decreased energy expenditure by $\mathrm{PD}$ patients compared to controls.[16]

Dysfunctional smell and taste perception, apathy, depression, slowed digestion and dysphagia all may individually, or in combination, affect weight. $[13,17,18,6]$ Additionally, the motor symptoms of PD may impact weight through direct and indirect effects on shopping, cooking, feeding, and socialization.[19, 20] Medications for PD symptoms may result in nausea and can be less effective when taken with food. Furthermore, inadequate absorption of food due to gastroparesis may result in a decreased availability of energy. Finally, cognitively impaired PD patients may not attend to consuming enough calories due to forgetfulness, especially when a caregiver is absent.[18, 6]

It is unclear whether reversing weight loss in PD patients will improve HRQL. Since PD patients typically return to the physician for routine follow up every 3 to 6 months, a practical recommendation may be to closely monitor weight at each visit, $[12,21]$ and to discuss modifiable factors such as proper nutrition and eating habits. This study did not address whether intervention to modify weight would affect HRQL. Whether weight gain can improve PD HRQL will be an important area for future research.

Strengths of the current study included the use of a large national outcomes database drawn from Centers of Excellence worldwide. Several limitations were encountered in this study and may have biased the results. The use of only experienced PD centers may have provided a sampling bias. The database lacked mood (depression) indices, beyond the 'emotion' dimension of the PDQ-39. Additionally, the NPF-QII dataset did not collect exact medications and dosages. Furthermore, the calculation of monthly weight change was averaged over the follow-up inter$\operatorname{val}(12 \pm 6$ months) which tends to minimize monthly fluctuations in weight and potential impact. A more narrow range may have yielded more accurate results but would have reduced the 
power of the study. Lastly, our study used absolute weight change rather than percent-weight change, which can have differing implications in individuals with varying body weight. It should be noted that direct causation could not be established due to the design of the study. Despite these limitations, the findings were robust and indicate that weight is a potential important factor to consider in the care of the PD patients.

\section{Conclusion}

This large real-world prospective database revealed that multiple factors were associated with interval weight loss in PD. The most unique aspect to the study was the quantification of a longitudinal relationship between weight loss and HRQL. Whether intervention to prevent weight loss can improve PD HRQL could be an important area for future research.

\section{Acknowledgments}

National Parkinson Foundation.

\section{Author Contributions}

Conceived and designed the experiments: UA NH MSO. Performed the experiments: UA YH YD SW PS MSO. Analyzed the data: UA YH YD NH IM NM CH PS SW MSO. Contributed reagents/materials/analysis tools: YH YD PS SW. Wrote the paper: UA YH YD NH IM NM CH PS SW MSO.

\section{References}

1. Nutt JG, Wooten GF. Clinical practice. Diagnosis and initial management of Parkinson's disease. The New England journal of medicine. 2005; 353(10):1021-7. doi: 10.1056/NEJMcp043908 PMID: 16148287

2. Chaudhuri KR, Schapira AH. Non-motor symptoms of Parkinson's disease: dopaminergic pathophysiology and treatment. Lancet neurology. 2009; 8(5):464-74. doi: 10.1016/S1474-4422(09)70068-7 PMID: 19375664

3. Chen H, Zhang SM, Hernan MA, Willett WC, Ascherio A. Weight loss in Parkinson's disease. Annals of neurology. 2003; 53(5):676-9. doi: 10.1002/ana.10577 PMID: 12731005

4. Delikanaki-Skaribas E, Trail M, Wong WW, Lai EC. Daily energy expenditure, physical activity, and weight loss in Parkinson's disease patients. Movement disorders: official journal of the Movement Disorder Society. 2009; 24(5):667-71. doi: 10.1002/mds.22372 PMID: 19117356

5. Lorefalt B, Ganowiak W, Palhagen S, Toss G, Unosson M, Granerus AK. Factors of importance for weight loss in elderly patients with Parkinson's disease. Acta neurologica Scandinavica. 2004; 110(3):180-7. doi: 10.1111/j.1600-0404.2004.00307.x PMID: 15285776

6. Uc EY, Struck LK, Rodnitzky RL, Zimmerman B, Dobson J, Evans WJ. Predictors of weight loss in Parkinson's disease. Movement disorders: official journal of the Movement Disorder Society. 2006; 21(7):930-6. doi: 10.1002/mds.20837 PMID: 16534756

7. Meguid MM, Fetissov SO, Varma M, Sato T, Zhang L, Laviano A et al. Hypothalamic dopamine and serotonin in the regulation of food intake. Nutrition. 2000; 16(10):843-57. PMID: 11054589

8. Beyer PL, Palarino MY, Michalek D, Busenbark K, Koller WC. Weight change and body composition in patients with Parkinson's disease. Journal of the American Dietetic Association. 1995; 95(9):979-83. doi: 10.1016/S0002-8223(95)00269-3 PMID: 7657912

9. Peto V, Jenkinson C, Fitzpatrick R, Greenhall R. The development and validation of a short measure of functioning and well being for individuals with Parkinson's disease. Quality of life research: an international journal of quality of life aspects of treatment, care and rehabilitation. 1995; 4(3):241-8.

10. Suchowersky O, Reich S, Perlmutter J, Zesiewicz T, Gronseth G, Weiner WJ et al. Practice Parameter: diagnosis and prognosis of new onset Parkinson disease (an evidence-based review): report of the Quality Standards Subcommittee of the American Academy of Neurology. Neurology. 2006; 66(7):968-75. doi: 10.1212/01.wnl.0000215437.80053.d0 PMID: 16606907 
11. Nirenberg MJ, Waters $C$. Compulsive eating and weight gain related to dopamine agonist use. Movement disorders: official journal of the Movement Disorder Society. 2006; 21(4):524-9. doi: 10.1002/ mds.20757

12. Bachmann CG, Trenkwalder C. Body weight in patients with Parkinson's disease. Movement disorders: official journal of the Movement Disorder Society. 2006; 21(11):1824-30. doi: 10.1002/mds.21068 PMID: 16958133

13. Barichella M, Cereda E, Pezzoli G. Major nutritional issues in the management of Parkinson's disease. Movement disorders: official journal of the Movement Disorder Society. 2009; 24(13):1881-92. doi: 10. 1002/mds.22705 PMID: 19691125

14. Levi S, Cox M, Lugon M, Hodkinson M, Tomkins A. Increased energy expenditure in Parkinson's disease. Bmj. 1990; 301(6763):1256-7. PMID: 2271826

15. Markus HS, Tomkins AM, Stern GM. Increased prevalence of undernutrition in Parkinson's disease and its relationship to clinical disease parameters. Journal of neural transmission Parkinson's disease and dementia section. 1993; 5(2):117-25. PMID: 8333907

16. Toth MJ, Fishman PS, Poehlman ET. Free-living daily energy expenditure in patients with Parkinson's disease. Neurology. 1997; 48(1):88-91. PMID: 9008499

17. Cersosimo MG, Benarroch EE. Neural control of the gastrointestinal tract: implications for Parkinson disease. Movement disorders: official journal of the Movement Disorder Society. 2008; 23(8):1065-75. doi: 10.1002/mds.22051 PMID: 18442139

18. Ross GW, Petrovitch H, Abbott RD, Tanner CM, Popper J, Masaki K et al. Association of olfactory dysfunction with risk for future Parkinson's disease. Annals of neurology. 2008; 63(2):167-73. doi: 10. 1002/ana.21291 PMID: 18067173

19. Abbott RA, Cox M, Markus H, Tomkins A. Diet, body size and micronutrient status in Parkinson's disease. European journal of clinical nutrition. 1992; 46(12):879-84. PMID: 1483417

20. Lorefat B, Ganowiak W, Wissing U, Granerus AK, Unosson M. Food habits and intake of nutrients in elderly patients with Parkinson's disease. Gerontology. 2006; 52(3):160-8. doi: 10.1159/000091825 PMID: 16645296

21. Keller HH, Gibbs AJ, Boudreau LD, Goy RE, Pattillo MS, Brown HM. Prevention of weight loss in dementia with comprehensive nutritional treatment. Journal of the American Geriatrics Society. 2003; 51(7):945-52. PMID: 12834514 